\title{
Predicting risk factors for rebleeding, infections, mortality following peptic ulcer bleeding in patients with cirrhosis and the impact of antibiotics prophylaxis at different clinical stages of the disease
}

Ming-Te Kuo ${ }^{1 \dagger}$, Shih-Cheng Yang ${ }^{1 \dagger}$, Lung-Sheng $\mathrm{Lu}^{1+}$, Chien-Ning Hsu², Yuan-Hung Kuo ${ }^{1}$, Chung-Huang Kuo ${ }^{1}$, Chih-Ming Liang ${ }^{1}$, Chung-Mou Kuo ${ }^{1}$, Cheng-Kun Wu' ${ }^{1}$ Wei-Chen Tai ${ }^{1,3^{*}}$ and Seng-Kee Chuah ${ }^{1,3}$

\begin{abstract}
Background: Infections in cirrhotic patients with upper gastrointestinal bleeding are a common event causing severe complication and mortality. This study aimed to identify risk factors that may predict rebleeding, bacterial infections, and the impact of antibiotic prophylaxis on mortality at different stages of cirrhosis following acute peptic ulcer bleeding (PUB).
\end{abstract}

Methods: A hospital-based retrospective cohort study was conducted on 235 cirrhotic patients with acute peptic ulcer hemorrhage who underwent therapeutic endoscopic procedures between January 2008 and January 2014 $(n=235)$; of these, 88 patients received prophylactic intravenous ceftriaxone (antibiotic group) and 147 patients did not (nil-antibiotic group). The recorded outcomes were length of hospital stay, bacterial infection, rebleeding, and in-hospital mortality.

Results: Forty-eight (20.4 \%) patients experienced ulcer rebleeding and 46 (19.6\%) developed bacterial infections. More patients suffered from infection and recurrent bleeding in the nil-antibiotic group than the antibiotic group ( $25.2 \%$ vs. $10.2 \%, p=0.005$ and $30.6 \%$ vs. $3.4 \% ; p<0.001$, respectively). The predictive risk factors for rebleeding were the Rockall score $(p=0.004)$, units of blood transfusion $(p=0.031)$, and no antibiotic prophylaxis $(p<0.001)$; for bacterial infections, they were the Child-Pugh score $(p=0.003)$, active alcoholism $(p=0.035)$, and no antibiotic prophylaxis $(p=0.009)$. Overall, $40(17 \%)$ patients died during hospitalization. The Rockall score and rebleeding were predictive factors for in-hospital mortality. In subgroup analysis, survival was significantly reduced in decompensated patients $(p=0.034)$.

(Continued on next page)

\footnotetext{
*Correspondence: luketai1019@gmail.com

${ }^{\dagger}$ Equal contributors

'Division of Hepatogastroenterology, Department of Internal Medicine, Kaohsiung Chang Gung Memorial Hospital, Chang Gung University College of Medicine, Kaohsiung, Taiwan

${ }^{3}$ Division of Hepatogastroenterology, Kaohsiung Chang Gang Memorial Hospital, 123 Ta-Pei Road, Niaosung Hsiang, Kaohsiung City 833, Taiwan Full list of author information is available at the end of the article
} to the Creative Commons license, and indicate if changes were made. The Creative Commons Public Domain Dedication waiver (http://creativecommons.org/publicdomain/zero/1.0/) applies to the data made available in this article, unless otherwise stated. 
(Continued from previous page)

Conclusions: This study suggests that antibiotic prophylaxis after endoscopic hemostasis for acute PUB prevented infections and reduced rebleeding events in cirrhotic patients. Antibiotic prophylaxis improved survival among decompensated cohort following PUB. The Rockall score and rebleeding were predictive risk factors for in-hospital mortality.

Keywords: Liver cirrhosis, Peptic ulcer bleeding, Antibiotic prophylaxis, Bacterial infections, Rebleeding, In-hospital mortality

\section{Background}

Upper gastrointestinal bleeding in cirrhotic patients is associated with an increased rate of failure to control bleeding and can result in mortality when bacterial infections occur $[1,2]$. This is evidenced by reports demonstrating that oral administration of non-absorbable antibiotics markedly reduces the incidence of bacterial infections in cirrhotic patients with gastrointestinal hemorrhage [3]. Patients with cirrhosis are at risk for both variceal and nonvariceal causes of upper gastrointestinal bleeding; however, only variceal bleeding has been widely studied. Nevertheless, there remains one third of nonvariceal upper gastrointestinal bleeding in cirrhotic patients due to peptic ulcers (PU) as reported in the literature [4]. In addition, bleeding PU have been associated with substantial morbidity and mortality in cirrhotic patients [5]. A multicenter prospective study from Italy reported that $10 \%$ of patients with cirrhosis rebleed and $15 \%$ of them died within 6 weeks after acute nonvariceal upper gastrointestinal bleeding [5].

Bacterial infection is more prone to initiate a systemic inflammatory response syndrome and increases the probability of death in patients with decompensated cirrhosis [6]. Generally, cirrhotic patients with PUB become infected and rebleed more frequently compared to noncirrhotic patients [4]. To our knowledge, prognosis after treating acute PUB has not been well studied in cirrhotic patients. Most studies on antibiotic prophylaxis in cirrhotic patients have focused on those with variceal hemorrhages [7-9]. Therefore, we conducted this study to identify the risk factors that predict re-bleeding, bacterial infections, mortality and the impact of antibiotic prophylaxis on death at different stages of cirrhosis following acute PUB.

\section{Methods}

\section{Ethics statements}

This retrospective chart review study was approved by both the Institutional Review Board and Ethics Committee of Chang Gung Memorial Hospital, Taiwan (IRB1035018B). The Ethics Committee waived the requirement for informed consent, and each patient's medical records was anonymized and de-identified prior to access. All patients provided their written inform consent before endoscopic interventions.

\section{Patient population}

This was a hospital-based retrospective cohort study including 426 cirrhotic eligible patients with PUB who underwent endoscopic interventions over a 6 year period between January 2008 and January 2014. The following patients were excluded from the study: (1) presence of any of the following signs of infection; fever $>38{ }^{\circ} \mathrm{C}$, white blood cell count $>10000 / \mathrm{mm}^{3}$, immature neutrophils $>500 / \mathrm{mm}^{3}$, a polymorphonuclear cell count in ascitic fluid $>250 / \mathrm{mm}^{3}, \geq 15$ leukocytes/field in the fresh urine sediment, or data compatible with pneumonia on the chest X-ray $(n=105),(2)$ allergy to cephalosporins $(n=2)$, (3) patients who received oral/parenteral antibiotics in the week prior to the procedure $(n=18),(4)$ a source of upper gastrointestinal bleeding other than PUB $(n=37)$, (5) patients who died within the first day after admission $(n=3)$, (6) patients who did not complete the in-hospital follow-up period $(n=26)$. A total of 235 patients were recruited into current study (male/female: 181/54; mean age: $62.2 \pm 13.6$ y). Those patients who received prophylactic intravenous infusion of ceftriaxone at $1 \mathrm{~g}$ per $12 \mathrm{~h}$ were classified as the antibiotic group $(n=88)$ in contrast to the control group who did not receive antibiotics $(n=147)$. The antibiotic group has been routine practice to prescribe prophylactic antibiotics to cirrhotic patients with gastrointestinal bleeding in our hospital since the year 2010 according to guidelines. On the other hand, those patients belonged to control group attended the hospital between January 2008 and December 2009. The antibiotic prophylaxis was given immediately after patients receiving endoscopic treatment and two sets of blood culture were obtained before administering the antibiotics even these patients did not encounter infections according to our hospital policy.

PUB was defined by the existence of signs of hematemesis, coffee ground vomitus, hematochezia, or melena and proven high-risk ulcers by endoscopy that were defined according to the Forrest classification [10]. High risk bleeding stigmata were referred for endoscopic view of active bleeding, visible vessels, or adherent clots. Patients' 
performance status was stratified according to the Rockall classification [11]. Blood transfusions were given to maintain hematocrit levels between $25 \%$ and $30 \%$. Patients with PUB were treated with intravenous high dose pantoprazole (80 mg intravenous bolus followed by $200 \mathrm{mg}$ continuous infusion for three days).

\section{Data collection and analysis}

The first authors abstracted following information from patient records (including written charts and electronic data sources). All patients had a complete medical assessment at initial hospital admission, including documentation of clinical, biochemical, and endoscopic factors that might contribute to rebleeding and mortality. The registered clinical variables were demographic data, clinical manifestations of bleeding, and the use of tobacco, alcohol, aspirin, clopidogrel, and non-steroidal antiinflammatory drugs (NSAID); co-morbidities such as diabetes mellitus, cardiovascular disease, stroke, end-stage renal disease, and chronic pulmonary disease were analyzed. Other clinical characteristics such as age, sex, and hemodynamic instability on admission and laboratory data such as white blood cell count, hemoglobin, platelet count, prothrombin time, serum creatinine, serum albumin, and total bilirubin were analyzed. The recorded endpoints were occurrence of infection, rebleeding, length of hospital stay, and in-hospital death.

\section{Definitions}

Time to endoscopy was defined as the time interval from admission via the emergency department to initial endoscopy, expressed in hours. The diagnosis of liver cirrhosis was based on clinical, laboratory, abdominal ultrasonographic, or histological findings [12]. Decompensated liver cirrhosis was used to describe patients complicated by ascites, jaundice (bilirubin level $>3.0 \mathrm{mg} / \mathrm{dl}$ ), and a history of identified varices or hepatic encephalopathy. Diagnosis of hepatitis $\mathrm{C}$ and $\mathrm{B}$ virus-related liver disease was determined with specific viral markers (HBsAg or anti-HCV). Alcohol-related liver disease was defined as daily alcohol consumption $>80 \mathrm{~g}$ in men and $>40 \mathrm{~g}$ in women for at least 10 years with negative viral, metabolic, and autoimmune markers [13]. Active alcoholism is defined as a continuing daily alcohol intake over $20 \mathrm{~g}$ in patients with alcoholic cirrhosis [13].

Rebleeding was defined as a new onset of hematemesis, melena, or both associated with tachycardia or hypovolemic shock or a decrease in serum hemoglobin level of $>2 \mathrm{~g} / \mathrm{dl}$ after successful endoscopic and pharmacological treatment and hemodynamic stability of at least a $24 \mathrm{~h}$ period of stable vital signs [14].

A diagnosis of bacteremia was made when the presence of viable bacteria in the blood and the clinical picture was consistent with this diagnosis. The diagnosis of spontaneous bacterial peritonitis was made when a positive culture of ascitic fluid was obtained with an ascitic fluid neutrophil count $\geq 250$ neutrophils/ $\mu$ l [15]. The diagnosis of pneumonia was made by clinical, radiological, and bacteriological data. The diagnosis of urinary tract infection was made when a positive culture of urine $\left(10^{5} \geq\right.$ colonies $\left./ \mathrm{ml}\right)$ was obtained with a urine neutrophil count $>10$ neutrophils/ $\mu$ l and associated clinical pictures.

\section{Statistical analysis}

All results were expressed as means \pm standard deviations for continuous variables and as relative frequencies or percentages for categorical variables. Distributions of continuous variables were analyzed by the $\mathrm{X}^{2}$ test, the Fisher's exact test, or the independent sample $t$-test, depending upon the type of data analyzed for the two groups where appropriate. The Kaplan-Meier analysis with the log-rank test was used to compare differences of death rates between the two groups. Variables were analyzed using the multivariate Cox proportional hazard model to determine independent predictive factors of infection, rebleeding, and mortality. All the variables in univariate analyses were analyzed in multivariate analyses. The results were expressed as a hazard ratio (HR) with $95 \%$ confidence intervals. All statistical analyses were performed using the SPSS v18.0 (Chicago, Illinois, USA). A $p$-value of $<0.05$ was considered statistically significant.

\section{Results}

All the patients' baseline characteristics are presented in Table 1. All patients underwent emergency endoscopy within $24 \mathrm{~h}$ of admission. The mean time from presentation of bleeding to endoscopy was $7.6 \pm 7 \mathrm{~h}$. One hundred and forty-six patients (62.1\%) suffered from bleeding gastric ulcers (GU), 73 (31.1 \%) from duodenal ulcers (DU), and 16 (6.8 \%) from both GU and DU. These ulcers had high risk stigmata of hemorrhage (Forrest Ia or Ib, $50.2 \%$ and Forrest II a or IIb, $38.7 \%)$. Among them, $57.4 \%$ were found to have varices without stigmata of recent hemorrhage [esophageal varices (EV): $51.4 \%$; gastric varices $(\mathrm{GV}): 0.9 \%$; combined EV/GV: $5.1 \%]$. The mean value of the Rockall scoring system was $4.7 \pm 1.6$ on admission.

The details of endoscopic interventions are also summarized in Table 1. Endoscopic intervention was performed on all patients with either monotherapy (65.5\%) or combination therapy (34.5\%). All patients received intravenous high dose proton pump inhibitors for 3 days after initial endoscopic hemostasis. The mean length of hospital stay was $14.9 \pm 11.6 \mathrm{~d}$. Patients in the antibiotic group had a similar hospital stay when compared with control patients $(p=0.282)$.

Overall, 48 (20.4\%) patients encountered rebleeding events. Rebleeding rate in the antibiotic group was 
Table 1 Clinical characteristics, endoscopic findings, and clinical outcome of cirrhotic patients with peptic ulcer bleeding $(n=235)$

\begin{tabular}{llll}
\hline Characteristics & $\begin{array}{l}\text { Antibiotic group } \\
(n=88)\end{array}$ & $\begin{array}{l}\text { Control group } \\
(n=147)\end{array}$ & $p$-value \\
\hline Age (y) & $61.8 \pm 15.2$ & $62.5 \pm 12.5$ & 0.699 \\
Male, $\mathrm{n}(\%)$ & $71(80.7)$ & $110(74.8)$ & 0.302 \\
$\begin{array}{l}\text { Etiology of liver cirrhosis } \\
\quad \text { Alcoholic, n (\%) }\end{array}$ & $24(27.3)$ & $37(25.2)$ & 0.722 \\
$\quad$ Viral hepatitis, n (\%) & $62(70.4)$ & $100(68.0)$ & 0.697 \\
$\quad$ Cryptogenic, n (\%) & $2(2.3)$ & $10(6.8)$ & 0.219 \\
Child-Pugh group & & &
\end{tabular}

$\begin{array}{ll}\text { A, n (\%) } & 40(45.5) \\ B, n(\%) & 31(35.2) \\ \text { C, n (\%) } & 17(19.3)\end{array}$

$68(46.3)$

0.905

Decompensated cirrhosis, n (\%) 44 (50.0)

$44(29.9)$

0.399

$35(23.8)$

\subsection{2}

Rockall score

$4.7 \pm 1.5$

$61(41.5)$

0.204

MELD score

$13.7 \pm 6.1$

$4.7 \pm 1.7$

Child-Pugh score

$6.9 \pm 1.5$

Gastroesophageal varices, n (\%) 51 (58.0)

Ulcer location

Gastric ulcer, n (\%)
Duodenal ulcer, n (\%)
Both, n (\%)

\section{Use of NSAID or aspirin/}

clopidogrel, n (\%)

Smoking, n (\%)

Active alcoholism, n (\%)

$55(62.5)$

27 (30.7)

$6(6.8)$

17 (19.3)

$14.1 \pm 6.0$

0.885

0.631

$7.1 \pm 1.7$

$84(57.1)$

\subsection{7}

0.903

Other comorbidities

$\begin{array}{llll}\text { Diabetes mellitus, n (\%) } & 31(35.2) & 40(27.2) & 0.25 \\ \text { Hypertension, n (\%) } & 39(44.3) & 54(36.7) & 0.31 \\ \text { Cardiovascular disease, n (\%) } & 8(9.1) & 7(4.8) & 0.299 \\ \text { Stroke, n (\%) } & 3(3.4) & 10(6.8) & 0.38 \\ \text { ESRD, n (\%) } & 3(3.4) & 13(8.8) & 0.47 \\ \text { COPD, n (\%) } & 8(9.1) & 7(4.8) & 0.26\end{array}$

Laboratory on admission

WBC $\left(10^{9} /\right)$

$\mathrm{Hb}(\mathrm{g} / \mathrm{dl})$

$\operatorname{PLT}\left(10^{9} / \mathrm{l}\right)$

Prothrombin time (s)

$33(37.5)$

$91(61.9)$

0.927

46 (31.3)

$10(6.8)$

20 (13.6)

1

Albumin ( $\mathrm{g} / \mathrm{dl}$ )

$63(42.9)$

0.245

31 (35.2)

55 (37.4)

0.419

0.736

0.251

0.311

0.299

0.381

0.471

0.269

Creatinine $(\mathrm{mg} / \mathrm{dl})$

Total bilirubin (mg/dl)

Clinical characteristics

Hypovolemic shock

on admission, $\mathrm{n}(\%)$

Blood units transfused (unit)
Table 1 Clinical characteristics, endoscopic findings, and clinical outcome of cirrhotic patients with peptic ulcer bleeding $(n=235)$ (Continued)

\begin{tabular}{|c|c|c|c|}
\hline \multicolumn{4}{|l|}{$\begin{array}{l}\text { Stigmata of recent } \\
\text { hemorrhage at ulcer }\end{array}$} \\
\hline Forrest la or lb ulcer, n (\%) & $44(50)$ & $74(50.3)$ & 0.960 \\
\hline Forrest lla or llb ulcer, n (\%) & $37(42)$ & $54(36.7)$ & 0.419 \\
\hline Forrest IIc ulcer, n (\%) & $7(8)$ & $19(13)$ & 0.240 \\
\hline $\begin{array}{l}\text { Time (h), bleeding to } \\
\text { endoscopic treatment }\end{array}$ & $6.9 \pm 6.7$ & $7.7 \pm 6.6$ & 0.382 \\
\hline \multicolumn{4}{|l|}{ Treatment } \\
\hline Epinephrine injection, n (\%) & $27(30.7)$ & $57(38.8)$ & 0.261 \\
\hline APC, n (\%) & $13(14.8)$ & $37(25.2)$ & 0.070 \\
\hline Hemoclipping, n (\%) & $14(15.9)$ & $6(4.1)$ & 0.003 \\
\hline Combined therapy, n (\%) & 34 (38.6) & $47(32.0)$ & 0.323 \\
\hline \multicolumn{4}{|l|}{ Outcomes } \\
\hline Hospital stay (d) & $15.7 \pm 13.1$ & $14.1 \pm 10.9$ & 0.282 \\
\hline Rebleeding, n (\%) & $3(3.4)$ & 45 (30.6) & $<0.001$ \\
\hline Infections, n (\%) & $9(10.2)$ & $37(25.2)$ & 0.005 \\
\hline In-hospital mortality, n (\%) & $12(13.6)$ & $28(19.0)$ & 0.112 \\
\hline $\begin{array}{l}\text { Failure to control } \\
\text { bleeding, } \mathrm{n}(\%)\end{array}$ & $1(1.1)$ & $7(4.8)$ & \\
\hline Sepsis, n (\%) & $3(3.4)$ & $7(4.8)$ & \\
\hline Multiple organ failure, n (\%) & $8(9.1)$ & $14(9.4)$ & \\
\hline
\end{tabular}

Abbreviations: $A P C$, argon plasma coagulation; $C O P D$, chronic obstructive pulmonary disease; $E S R D$, end-stage renal disease; $E V$, esophageal varices; GOV, Gastroesophageal varices; IGV, Isolated gastric varices; MELD, Model for End-Stage Liver Disease; NSAID, nonsteroidal anti-inflammatory drug; $W B C$, white blood cells; $H b$, hemoglobin; $P L T$, platelet count; $P T$, prothrombin time

significantly lower than the control group (3.4\% vs $30.6 \%$; p <0.001; Table 1). On multivariate analysis, the risk factors for rebleeding were higher rockall score $[\mathrm{HR}=$ 1.069; $95 \%$ confidence interval $(\mathrm{CI})=1.022-1.120 ; p=$ $0.004]$ and more units of blood transfusion $(\mathrm{HR}=1.019$; $95 \% \mathrm{CI}=1.002-1.037 ; p=0.031$ ). Antibiotic prophylaxis has a protective role for rebleeding in these patients $(\mathrm{HR}=0.082 ; 95 \% \mathrm{CI}=0.025-0.267 ; \mathrm{p}<0.001)$ (Table 2). It is interesting that time to endoscopy, high risk bleeding stigmata, and endoscopic therapeutic methods were not associated with rebleeding rate (Table 2).

Bacterial infections were documented in 46 patients (19.6\%); more patients were infected in the control group (10.2 \% vs. $25.2 \%$; $p=0.005)$. Infections were confirmed for 9 patients in the antibiotics group (bacteremia in 4, spontaneous bacterial peritonitis in 2, pneumonia in 1 and urinary tract infection in 2). On the other hand, 37 bacterial infections were proven in the control group (bacteremia in 17, pneumonia in 4, spontaneous bacterial peritonitis in 10, and urinary tract infections in 6). The causative organisms of bacteremia were gram- 
Table 2 Univariate and multivariate analysis of potential risk factors for rebleeding in patients with peptic ulcer bleeding after endoscopic treatment

\begin{tabular}{|c|c|c|c|c|}
\hline \multirow[t]{2}{*}{ Variable } & \multicolumn{2}{|l|}{ Univariate analysis } & \multicolumn{2}{|l|}{$\underline{\text { Multivariate analysis }}$} \\
\hline & Hazard ratio $(95 \% \mathrm{Cl})$ & $p$-value & Hazard ratio $(95 \% \mathrm{Cl})$ & $p$-value \\
\hline Age & $0.991(0.971-1.012)$ & 0.383 & & \\
\hline Male gender & $2.882(1.141-7.283)$ & 0.025 & 2.639 (0.968-7.198) & 0.058 \\
\hline \multicolumn{5}{|l|}{ Etiology of liver cirrhosis } \\
\hline Alcoholic & $1.001(0.521-1.924)$ & 0.998 & & \\
\hline Viral hepatitis & $1.086(0.790-1.494)$ & 0.610 & & \\
\hline Cryptogenic & $0.715(0.369-1.384)$ & 0.319 & & \\
\hline Decompensated cirrhosis & $1.559(0.881-2.758)$ & 0.127 & & \\
\hline Rockall score & $1.071(1.031-1.111)$ & $<0.001$ & $1.069(1.022-1.120)$ & 0.004 \\
\hline MELD score & $1.141(0.964-1.351)$ & 0.126 & & \\
\hline Child-Pugh score & $1.321(1.126-1.548)$ & 0.001 & $1.128(0.923-1.378)$ & 0.238 \\
\hline \multicolumn{5}{|l|}{ Other comorbidities } \\
\hline Diabetes mellitus & $1.244(0.689-2.249)$ & 0.469 & & \\
\hline Hypertension & $0.688(0.378-1.255)$ & 0.233 & & \\
\hline CVD & $0.948(0.230-3.912)$ & 0.941 & & \\
\hline Stroke & $1.360(0.422-4.382)$ & 0.607 & & \\
\hline ESRD & $1.246(0.448-3.470)$ & 0.673 & & \\
\hline COPD & $0.495(0.145-1.683)$ & 0.260 & & \\
\hline Use of NSAID or aspirin & $1.479(0.586-3.734)$ & 0.408 & & \\
\hline Smoking & $1.362(0.771-2.405)$ & 0.287 & & \\
\hline Active alcoholism & $1.585(0.898-2.799)$ & 0.112 & & \\
\hline WBC $\left(10^{9} / \mathrm{l}\right)$ & 1 & 0.886 & & \\
\hline $\mathrm{Hb}(\mathrm{g} / \mathrm{dl})$ & $0.990(0.869-1.128)$ & 0.880 & & \\
\hline $\operatorname{PLT}\left(10^{9} / \mathrm{l}\right)$ & 0.997 (0.992-1.002) & 0.260 & & \\
\hline PT (s) & $1.286(0.749-2.207)$ & 0.362 & & \\
\hline Creatinine (mg/dl) & $1.063(0.966-1.171)$ & 0.213 & & \\
\hline Albumin $(g / l)$ & $0.635(0.402-1.005)$ & 0.053 & & \\
\hline Total bilirubin & $1.068(1.012-1.128)$ & 0.017 & $1.042(0.967-1.122)$ & 0.277 \\
\hline Blood units transfused & $1.032(1.015-1.050)$ & $<0.001$ & $1.019(1.002-1.037)$ & 0.031 \\
\hline Hypovolemic shock on admission & $1.143(0.453-2.887)$ & 0.777 & & \\
\hline High risk bleeding stigmata at ulcer base & $1.103(0.407-2.989)$ & 0.848 & & \\
\hline Time (h), bleeding to endoscopic treatment & $0.989(0.959-1.039)$ & 0.940 & & \\
\hline Combined treatment of endoscopic hemostasis & $0.941(0.521-1.700)$ & 0.840 & & \\
\hline Bacterial infection & $1.974(1.081-3.607)$ & 0.027 & $1.012(0.527-1.941)$ & 0.972 \\
\hline Antibiotic prophylaxis & $0.090(0.028-0.291)$ & $<0.001$ & $0.082(0.025-0.267)$ & $<0.001$ \\
\hline
\end{tabular}

Abbreviations: $C l$, confidence interval; COPD, chronic obstructive pulmonary disease; $C V D$, Cardiovascular disease; ESRD, end-stage renal disease; MELD, Model for End-Stage Liver Disease; NSAID, nonsteroidal anti-inflammatory drug; WBC, white blood cells; $H b$, hemoglobin; $P L T$, platelet count; $P T$, prothrombin time

negative bacilli in 15 patients (Klebsiella pneumoniae in 7; Escherichia coli in 6; Pseudomonas aeruginosa in 2) and gram-positive cocci in 6 patients (Streptococcus pneumoniae in 4; Staphylococcus aureus in 1; Enterococcus faecalis in 1). The risk factors for bacterial infection were higher Child-Pugh score $(\mathrm{HR}=1.251 ; 95 \% \mathrm{CI}=1.080$ -
1.449; $p=0.003)$ and active alcoholism $(\mathrm{HR}=1.882 ; 95 \%$ $\mathrm{CI}=1.045-3.388 ; p=0.035)$. Antibiotic prophylaxis played a significant role to prevent infections $(\mathrm{HR}=0.377 ; 95 \%$ $\mathrm{CI}=0.180-0.786 ; p=0.009$ ) (Table 3 ).

In-hospital death occurred in 40 patients (17\%). Causes of death were hypovolemic shock in 8 patients ( 1 in the 
Table 3 Univariate and multivariate analysis of potential risk factors for infection in patients with peptic ulcer bleeding after endoscopic treatment

\begin{tabular}{|c|c|c|c|c|}
\hline \multirow[t]{2}{*}{ Variable } & \multicolumn{2}{|l|}{ Univariate analysis } & \multicolumn{2}{|l|}{$\underline{\text { Multivariate analysis }}$} \\
\hline & Hazard ratio $(95 \% \mathrm{Cl})$ & $p$-value & Hazard ratio $(95 \% \mathrm{Cl})$ & $p$-value \\
\hline Age & $0.989(0.968-1.010)$ & 0.286 & & \\
\hline Male gender & $1.171(0.580-2.363)$ & 0.660 & & \\
\hline \multicolumn{5}{|l|}{ Etiology of liver cirrhosis } \\
\hline Alcoholic & $1.869(1.015-3.441)$ & 0.045 & $1.842(0.899-3.774)$ & 0.095 \\
\hline Viral hepatitis & $0.769(0.572-1.035)$ & 0.083 & & \\
\hline Cryptogenic & $0.933(0.582-1.498)$ & 0.775 & & \\
\hline Decompensated cirrhosis & $2.339(1.273-4.298)$ & 0.006 & $1.688(0.707-4.043)$ & 0.239 \\
\hline Rockall score & $1.275(1.048-1.552)$ & 0.015 & $1.141(0.944-1.381)$ & 0.173 \\
\hline MELD score & $1.061(1.022-1.100)$ & 0.002 & $1.048(0.988-1.111)$ & 0.121 \\
\hline Child-Pugh score & $1.289(1.113-1.493)$ & 0.001 & $1.251(1.080-1.449)$ & 0.003 \\
\hline \multicolumn{5}{|l|}{ Other comorbidities } \\
\hline Diabetes mellitus & $1.272(0.693-2.338)$ & 0.437 & & \\
\hline Hypertension & $0.971(0.537-1.757)$ & 0.923 & & \\
\hline CVD & $0.483(0.066-3.510)$ & 0.472 & & \\
\hline Stroke & $0.412(0.057-2.990)$ & 0.380 & & \\
\hline ESRD & $1.661(0.402-6.860)$ & 0.483 & & \\
\hline COPD & $1.981(0.837-4.686)$ & 0.120 & & \\
\hline Use of NSAID or aspirin & $0.127(0.018-0.925)$ & 0.042 & $0.182(0.025-1.336)$ & 0.094 \\
\hline Smoking & $1.301(0.725-2.335)$ & 0.378 & & \\
\hline Active alcoholism & $1.924(1.075-3.446)$ & 0.028 & $1.616(0.789-3.310)$ & 0.190 \\
\hline WBC $\left(10^{9} / \mathrm{l}\right)$ & 1 & 0.804 & & \\
\hline $\mathrm{Hb}(\mathrm{g} / \mathrm{dl})$ & $0.899(0.775-1.019)$ & 0.090 & & \\
\hline $\operatorname{PLT}\left(10^{9} / l\right)$ & $0.996(0.991-1.001)$ & 0.160 & & \\
\hline PT (second) & $1.290(0.781-2.129)$ & 0.319 & & \\
\hline Creatinine (mg/dl) & $0.958(0.830-1.106)$ & 0.562 & & \\
\hline Albumin $(g / l)$ & $0.921(0.592-1.434)$ & 0.716 & & \\
\hline Total bilirubin & $1.071(1.019-1.127)$ & 0.008 & $1.006(0.929-1.088)$ & 0.887 \\
\hline Blood units transfused & $1.016(0.993-1.0140$ & 0.181 & & \\
\hline Hypovolemic shock on admission & $1.328(0.525-3.362)$ & 0.550 & & \\
\hline High risk bleeding stigmata at ulcer base & $2.830(0.686-11.68)$ & 0.150 & & \\
\hline Time (h), bleeding to endoscopic treatment & $1.026(0.997-1.055)$ & 0.077 & & \\
\hline Combined treatment of endoscopic hemostasis & $0693(0.385-1.248)$ & 0.222 & & \\
\hline Recurrent bleeding & $1.974(1.075-3.623)$ & 0.028 & $1.601(0.781-3.282)$ & 0.906 \\
\hline Antibiotic prophylaxis & $0.346(0.167-0.719)$ & 0.004 & $0.377(0.180-0.786)$ & 0.009 \\
\hline
\end{tabular}

Abbreviations: $\mathrm{Cl}$, confidence interval; COPD, chronic obstructive pulmonary disease; CVD, Cardiovascular disease; ESRD, end-stage renal disease; MELD, Model for End-Stage Liver Disease; NSAID, nonsteroidal anti-inflammatory drug; WBC, white blood cells; $H b$, hemoglobin; $P L T$, platelet count; $P T$, prothrombin time

antibiotic group and 7 in control group), sepsis in 10 patients ( 3 and 7 patients, respectively) and multiple organ failure in 22 patients ( 8 and 14 patients, respectively). There was no significant difference in mortality during hospitalization between patients treated with intravenous ceftriaxone $(n=12,13.6 \%)$ and those in the control group ( $n=28,19 \%)$. The observed survival was virtually identical for both groups $(p=0.112$; Table 1$)$. The results of the univariate and multivariate analyses for independent risks of death after acute PUB are summarized in Table 4. The results of univariate analysis showed that decompensated cirrhosis, total bilirubin level, Rockall score, 
Table 4 Univariate and multivariate analysis of potential risk factors for death in patients with peptic ulcer bleeding after endoscopic treatment

\begin{tabular}{|c|c|c|c|c|}
\hline \multirow[t]{2}{*}{ Variable } & \multicolumn{2}{|l|}{ Univariate analysis } & \multicolumn{2}{|l|}{ Multivariate analysis } \\
\hline & Hazard ratio $(95 \% \mathrm{Cl})$ & $p$-value & Hazard ratio $(95 \% \mathrm{Cl})$ & $p$-value \\
\hline Age & $0.981(0.958-1.004)$ & 0.112 & & \\
\hline Male gender & $2.046(0.902-4.639)$ & 0.087 & & \\
\hline \multicolumn{5}{|l|}{ Etiology of liver cirrhosis } \\
\hline Alcoholic & $1.188(0.577-2.455)$ & 0.641 & & \\
\hline Viral hepatitis & $1.023(0.722-1.488)$ & 0.887 & & \\
\hline Cryptogenic & $0.713(0.366-1.392)$ & 0.322 & & \\
\hline Decompensated cirrhosis & $2.112(1.014-4.402)$ & 0.046 & $1.259(0.497-3.188)$ & 0.627 \\
\hline Rockall score & $1.638(1.327-2.022)$ & $<0.001$ & $1.884(1.477-2.404)$ & $<0.001$ \\
\hline MELD score & 1.049 (1.007-1.093) & 0.023 & $0.945(0.875-1.020)$ & 0.417 \\
\hline Child-Pugh score & $1.321(1.126-1.548)$ & 0.001 & $1.252(0.915-1.712)$ & 0.163 \\
\hline \multicolumn{5}{|l|}{ Other comorbidities } \\
\hline Diabetes mellitus & $1.163(0.825-1.639)$ & 0.388 & & \\
\hline Hypertension & $0.826(0.437-1.562)$ & 0.555 & & \\
\hline CVD & $1.656(0.396-6.924)$ & 0.490 & & \\
\hline Stroke & $0.830(0.194-3.542)$ & 0.801 & & \\
\hline ESRD & $1.462(0.445-4.808)$ & 0.532 & & \\
\hline COPD & $0.495(0.145-1.683)$ & 0.260 & & \\
\hline Use of NSAID or aspirin & $1.381(0.765-2.493)$ & 0.284 & & \\
\hline Smoking & $1.040(0.739-1.465)$ & 0.820 & & \\
\hline Active alcoholism & $1.453(0.771-2.739)$ & 0.248 & & \\
\hline WBC $\left(10^{9} /\right)$ & 1 & 0.737 & & \\
\hline $\mathrm{Hb}(\mathrm{g} / \mathrm{dl})$ & $0.941(0.809-1.095)$ & 0.431 & & \\
\hline $\operatorname{PLT}\left(10^{9} / \mathrm{l}\right)$ & $0.997(0.992-1.002)$ & 0.211 & & \\
\hline PT (second) & $1.355(0.823-2.232)$ & 0.232 & & \\
\hline Creatinine (mg/dl) & $1.003(0.857-1.174)$ & 0.972 & & \\
\hline Albumin (g/l) & $0.906(0.555-1.479)$ & 0.692 & & \\
\hline Total bilirubin & $1.056(1.004-1.111)$ & 0.033 & $0.987(0.916-1.063)$ & 0.731 \\
\hline Blood units transfused & $0.989(0.961-1.018)$ & 0.468 & & \\
\hline Hypovolemic shock on admission & $1.184(0.420-3.341)$ & 0.750 & & \\
\hline High risk bleeding stigmata at ulcer base & $1.103(0.407-2.989)$ & 0.848 & & \\
\hline Time (h), bleeding to endoscopic treatment & $0.972(0.936-1.010)$ & 0.143 & & \\
\hline Combined treatment of endoscopic hemostasis & $0.966(0.485-1.926)$ & 0.922 & & \\
\hline Bacterial infection & $1.770(0.945-3.318)$ & 0.075 & & \\
\hline Recurrent bleeding & $2.412(1.292-4.501)$ & 0.006 & $2.796(1.473-5.306)$ & 0.002 \\
\hline Antibiotic prophylaxis & $0.582(0.293-1.151)$ & 0.121 & & \\
\hline
\end{tabular}

Abbreviations: $C l$, confidence interval; COPD, chronic obstructive pulmonary disease; $C V D$, Cardiovascular disease; ESRD, end-stage renal disease; $M E L D$, Model for End-Stage Liver Disease; NSAID, nonsteroidal anti-inflammatory drug; WBC, white blood cells; Hb, hemoglobin; PLT, platelet count; PT, prothrombin time

MELD score, Child-Pugh score, bacterial infection, and recurrent bleeding were associated with an increased risk of death. In multivariate analysis, the in-hospital mortality was remarkably dependent on Rockall score $(\mathrm{HR}=$ 1.884; $95 \% \mathrm{CI}=1.477-2.404 ; \mathrm{p}<0.001$ ) and recurrent bleeding $(\mathrm{HR}=2.796 ; 95 \% \mathrm{CI}=1.473-5.306 ; p=0.002)$.

\section{Subgroup analytical result for compensated and decompensated patients}

By using the Kaplan-Meier approach, the administration of prophylactic antibiotics was not associated with significant differences in in-hospital mortality between our cohort ( $13.6 \%$ vs. $19 \%, p=0.112$ by log-rank test; 
Fig. 1). On the other hand, we observed that the in-hospital mortality was $28.6 \%$ in patients with decompensated cirrhosis and $7.7 \%$ in patients with compensated cirrhosis $(\mathrm{p}<0.001)$ following acute PUB. Because the lack of a beneficial effect may be related to the severity of liver disease, we conducted a sub-analysis on the basis of liver decompensation among these cirrhotic patients. The observed in-hospital mortality was virtually identical for both groups of patients with baseline compensated cirrhosis (93.2 \% vs. $91.9 \%, p=0.830$ by log-rank test; Fig. 2). However, the administration of prophylactic antibiotics showed significantly reduction of in-hospital mortality of patients with baseline decompensated cirrhosis compared to those without antibiotic prophylaxis (79.5 \% vs. $65.6 \%, p=0.034$ by log-rank test) after subgroup analysis (Fig. 3). The predictive risk factor associated with in-hospital death among decompensated cirrhotic patients was Rockall score (HR, 1.623; 95 \% CI, $1.204-2.187 ; p=0.001)$. Antibiotic prophylaxis has a protective role for in-hopital death in these patients (HR, 0.395; $95 \%$ CI, 0.173-0.899; $p=0.027)$. For compensated cirrhotic patient, Rockall score (HR, 1.633; 95 \% CI, 1.1032.417; $p=0.014)$ and recurrent bleeding ( $\mathrm{HR}, 3.684 ; 95 \%$ CI, $1.040-13.05 ; p=0.044)$ were the predictive factors associated with in-hospital death (Table 5).

\section{Discussion}

Multiple factors contribute to the infection of cirrhotic patients with upper gastrointestinal bleeding. Cirrhotic patients usually have host defense defects against bacterial infection [16]. Furthermore, hypovolemia has been reported to increase intestinal bacterial translocation and depress reticuloendothelial system activity [17, 18]. In addition, patients with decompensated cirrhosis have more frequent episodes of infection than those with compensated liver disease [19]. Bacterial infection is associated with failure to control bleeding and patients with recurrent bleeding episodes [20]. An increase in portal pressure and changes in hemostasis induced by infection have been suggested as possible mechanisms $[1,21]$. It has been suggested that intestinal bacterial translocation plays an important role in the pathogenesis of many infections. A meta-analysis of 12 trials comprising a total of 1241 patients with cirrhosis and gastrointestinal bleeding demonstrated that antibiotic prophylaxis significantly decreased the incidence of bacterial infections, re-bleeding, length of hospitalization, and mortality [22]. Currently, antibiotic prophylaxis is the standard care for cirrhotic patients with acute variceal bleeding but the prescription of antibiotic prophylaxis in cirrhotic patient with PUB has not been well documented. In order to address this particular issue in cirrhotic patients with PUB, we conducted this hospital-based cohort study for further clarification.

The results of the current study proved the benefit of antibiotic prophylaxis for bleeding peptic ulcers in cirrhotic patients with only 9 of the 88 patients (10.8\%) included in the antibiotic group developing bacterial infections during hospitalization. On the other hand, 37 of the 147 patients (25.2 \%) in the control group suffered from a greater number of infectious events. In the multivariate

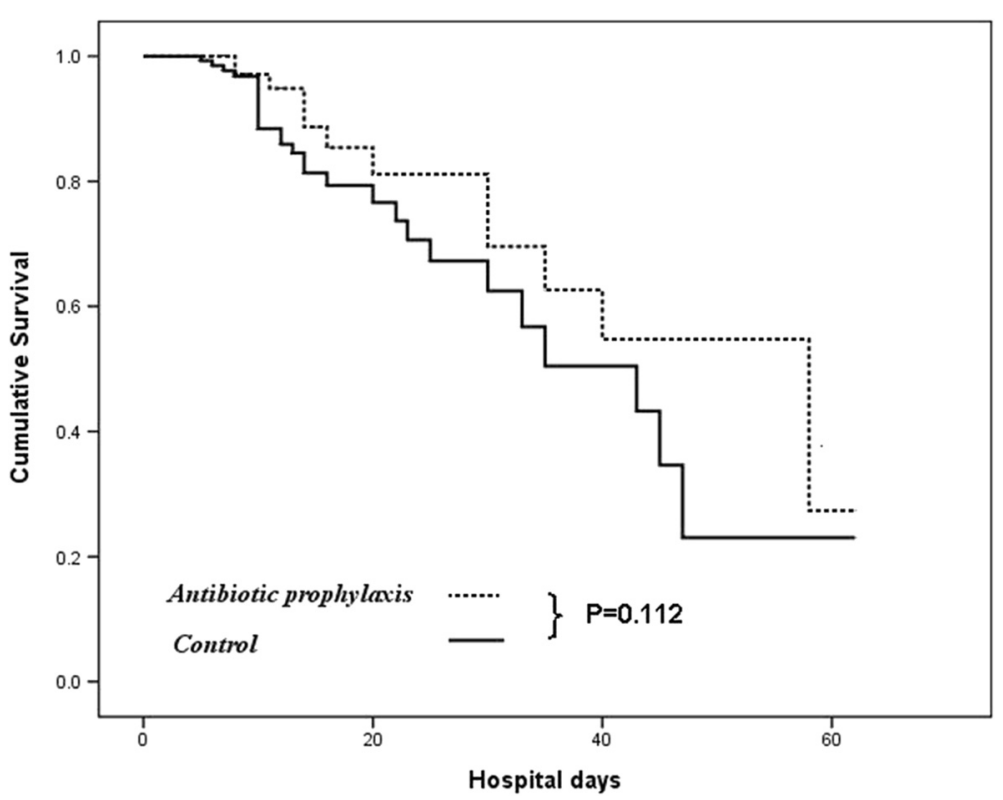

Fig. 1 Actuarial probability of remaining survival in all cirrhotic patients after endoscopic interventions for the ceftriaxone group (antibiotic prophylaxis group) and the nil- antibiotic prophylaxis group (control group) 


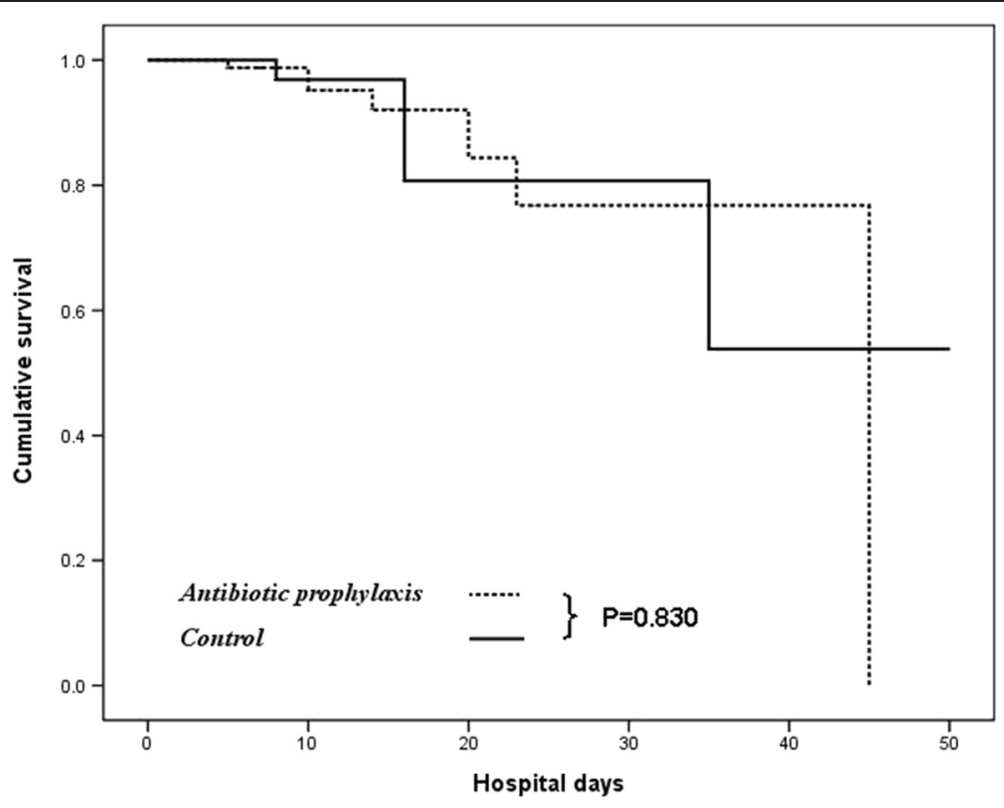

Fig. 2 Actuarial probability of remaining survival at different stages of cirrhotic patients (compensated liver cirrhosis). There was a similar probability of survival between compensated patients who were prescribed with intravenous ceftriaxone and those without antibiotic prophylaxis $(p=0.830$ by log-rank test)

analysis, higher Child-Pugh score, active alcoholism, and no antibiotic prophylaxis were significant predictors of infection development. Bacterial infections have been observed more frequently in Child-Pugh's $C$ patients than in those with Child-Pugh's A or B [23] and active alcoholism can increase host susceptibility to bacterial infection, probably owing to the immunocompromised status following liver decompensation [24]. The bottom line is, the most common pathogens cultured in cirrhotic patients are gram-negative bacilli $[15,20]$. The hypothesis

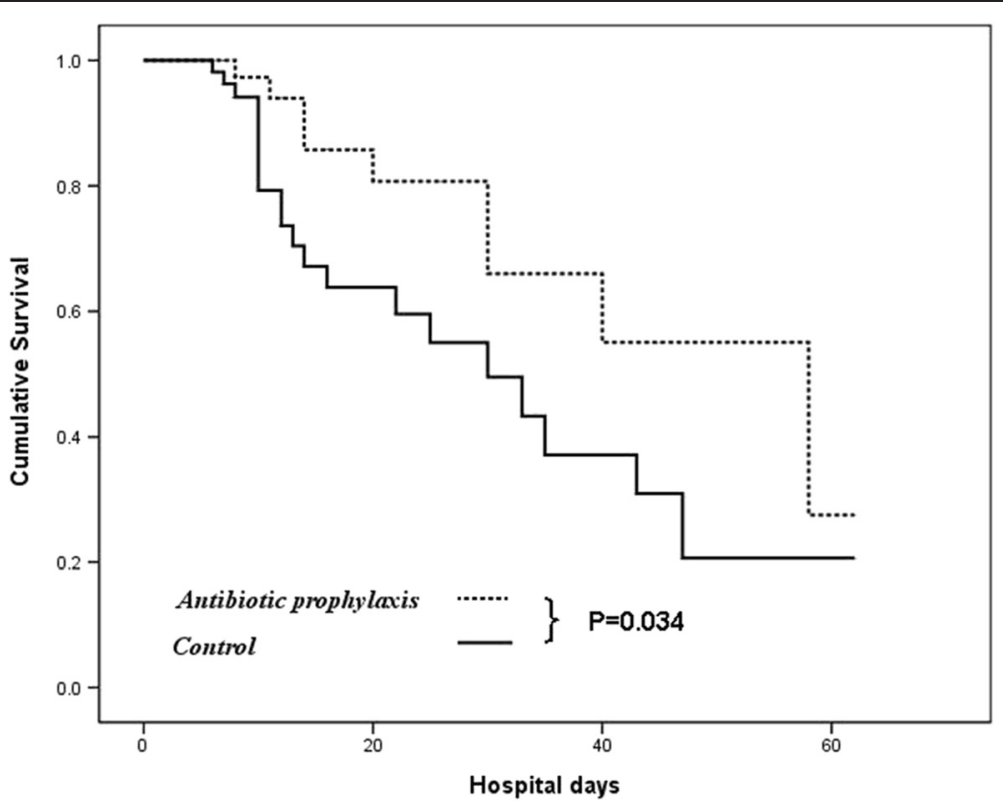

Fig. 3 Actuarial probability of remaining survival at different stages of cirrhotic patients (decompensated liver cirrhosis). A significantly higher probability of remaining survival was observed in those who were prescribed with intravenous ceftriaxone than those without antibiotic prophylaxis ( $p=0.034$ by log-rank test) 
Table 5 Univariate and multivariate analysis of potential risk factors for mortality with peptic ulcer bleeding after endoscopic treatment at different clinical stages of cirrhotic patients (subgroup analysis)

\begin{tabular}{|c|c|c|c|c|c|c|}
\hline \multirow[t]{3}{*}{ Variable } & \multicolumn{3}{|c|}{ Compensated liver cirrhosis $(N=130)$} & \multicolumn{3}{|c|}{ Decompensated liver cirrhosis $(N=105)$} \\
\hline & \multirow{2}{*}{$\begin{array}{l}\text { Univariate analysis } \\
p \text {-value }\end{array}$} & \multicolumn{2}{|l|}{ Multivariate analysis } & \multirow{2}{*}{$\begin{array}{l}\text { Univariate analysis } \\
p \text {-value }\end{array}$} & \multicolumn{2}{|l|}{ Multivariate analysis } \\
\hline & & Hazard ratio $(95 \% \mathrm{Cl})$ & $p$-value & & Hazard ratio $(95 \% \mathrm{Cl})$ & $p$-value \\
\hline Age & 0.753 & & & 0.115 & & \\
\hline Male gender & 0.140 & & & 0.351 & & \\
\hline \multicolumn{7}{|l|}{ Etiology of liver cirrhosis } \\
\hline Alcoholic & 0.136 & & & 0.639 & & \\
\hline Viral hepatitis & 0.281 & & & 0.422 & & \\
\hline Cryptogenic & 0.644 & & & 0.482 & & \\
\hline Rockall score & 0.014 & $1.633(1.103-2.417)$ & 0.014 & 0.001 & $1.623(1.204-2.187)$ & 0.001 \\
\hline MELD score & 0.713 & & & 0.151 & & \\
\hline Child-Pugh score & 0.236 & & & 0.013 & $1.088(0.855-1.384)$ & 0.492 \\
\hline \multicolumn{7}{|l|}{ Other comorbidities } \\
\hline Diabetes mellitus & 0.394 & & & 0.477 & & \\
\hline Hypertension & 0.352 & & & 0.831 & & \\
\hline CVD & 0.729 & & & 0.195 & & \\
\hline Stroke & 0.168 & & & 0.450 & & \\
\hline ESRD & 0.986 & & & 0.128 & & \\
\hline COPD & 0.584 & & & 0.157 & & \\
\hline Use of NSAID or aspirin & 0.386 & & & 0.128 & & \\
\hline Smoking & 0.629 & & & 0.975 & & \\
\hline Active alcoholism & 0.533 & & & 0.177 & & \\
\hline WBC $\left(10^{9} / l\right)$ & 0.428 & & & 0.880 & & \\
\hline $\mathrm{Hb}(\mathrm{g} / \mathrm{dl})$ & 0.921 & & & 0.463 & & \\
\hline $\operatorname{PLT}\left(10^{9} / \mathrm{l}\right)$ & 0.796 & & & 0.375 & & \\
\hline PT (second) & 0.898 & & & 0.597 & & \\
\hline Creatinine (mg/dl) & 0.766 & & & 0.869 & & \\
\hline Albumin (g/l) & 0.517 & & & 0.955 & & \\
\hline Total bilirubin & 0.450 & & & 0.167 & & \\
\hline Blood units transfused & 0.794 & & & 0.309 & & \\
\hline Hypovolemic shock on admission & 0.396 & & & 0.751 & & \\
\hline High risk bleeding stigmata at ulcer base & 0.593 & & & 0.676 & & \\
\hline Time (h), bleeding to endoscopic treatment & 0.734 & & & 0.085 & & \\
\hline $\begin{array}{l}\text { Combined treatment of endoscopic } \\
\text { hemostasis }\end{array}$ & 0.229 & & & 0.538 & & \\
\hline Bacterial infection & 0.327 & & & 0.224 & & \\
\hline Recurrent bleeding & 0.043 & $3.684(1.040-13.05)$ & 0.044 & 0.096 & & \\
\hline Antibiotic prophylaxis & 0.830 & & & 0.042 & $0.395(0.173-0.899)$ & 0.027 \\
\hline
\end{tabular}

Abbreviations: $C l$, confidence interval; COPD, chronic obstructive pulmonary disease; CVD, Cardiovascular disease; ESRD, end-stage renal disease; $M E L D$, Model for End-Stage Liver Disease; NSAID, nonsteroidal anti-inflammatory drug; WBC, white blood cells; Hb, hemoglobin; PLT, platelet count; PT, prothrombin time

behind this could be the onset of infection by intestinal bacterial translocation. In our study, gram-positive cocci accounted for $28.6 \%$ of infections. This was probably because cirrhotic patients with upper gastrointestinal bleeding tended to receive multiple sessions of diagnostic and therapeutic invasive procedures, which in turn resulted in infections with gram-positive cocci $[25,26]$.

Overall, the ulcer rebleeding rate observed in the current study (22.3\%) which was higher than those reported in non-cirrhotic patients (3.2\%) [27] or in cirrhotic 
patients (7\%) [28]. The higher rebleeding events may be explained by the occurrence of a greater number of high risk bleeding stigmata identified in all of our patients with PUB (Forrest Ia, Ib: 51.9 \% and Forrest IIa, IIb: $42.4 \%$ ) compared with those reported by the Marmo and Rudler groups [27, 28]. However, we observed that rebleeding and mortality do not seem to be associated with time to endoscopy. Although patients in the present study received endoscopy within $24 \mathrm{~h}$, mortality remains high compared to patients without cirrhosis in the literature [29]. The predictors for rebleeding were: a higher Rockall score, more units of blood transfused, and no antibiotic prophylaxis. Again, current study proved the importance of administrating prophylactic antibiotics is crucial in preventing rebleeding in cirrhotic patients. Although presence of high risk bleeding stigmata is well-established risk factor for peptic ulcer bleeding, we didn't find significantly correlation with rebleeding. Several factors might contribute to the discrepant results. First, it is possible that this retrospective analysis depended heavily on the completeness of the medical records and bias could exist. However, we reviewed endoscopic images or videos to determine the severity of the ulcer involved if uncertain chart description of ulcer morphology was encountered. Second, it was probably due to most ulcers belonged to high stigmata (Forrest Ia, Ib: 50.2 \% and Forrest IIa, IIb: 38.7 \%) in all of our enrolled patients. In fact, current guidelines do not recommend hemostatic therapy for patients with lowrisk stigmata, such as those ulcers with a clean base (Forrest III) or ulcers with flat spots (Forrest IIc), and even recommend early discharge in selected patients with a low risk of rebleeding after endoscopic evaluation.

However, our study failed to show a significant difference in mortality or reduced length of hospital stay between patients who received prophylactic antibiotics and the control group. Probably, other factors such as requirement for mechanical ventilation, length of stay in intensive care unit, multiple organ dysfunction, and underlying comorbidities contributed to longer hospital stays and could be important determinants other than prophylactic antibiotic administration. Our study also clearly showed the increased mortality of patients with decompensated cirrhosis compared to those with compensated cirrhosis, with a quadrupling tripling of mortality risk (28.6\% vs. $7.7 \%)$. Moreover, there was still an increased mortality of patients with decompensated cirrhosis compared with compensated cirrhosis even among patients with antibiotic prophylaxis (20.5\% vs. $6.8 \%$ ). Overall, the mortality rate in this study was $17 \%$. This was higher than those reported in previous studies of PUB [30]. This was not surprising because $44.7 \%$ of our patients had decompensated cirrhosis. Patients with cirrhosis who develop PUB were at increased risk of mortality, and the risk appeared to increase further as patients progressed from compensated to decompensated cirrhosis. This could be due to the fact that patients with decompensated cirrhosis have an increased risk of developing sepsis, multiple organ failure, and death [31]. To our knowledge, our study was the first to compare the outcomes in cirrhotic patients with or without liver compensation who received antibiotic prophylaxis for PUB. Because no beneficial effect of antibiotic prophylaxis may be attributed to the severity of liver disease, we decided to perform a subgroup analysis on the basis of liver compensation by dividing our patients into 2 subgroups. Our results revealed an important message that intravenous ceftriaxone is only of benefit in reducing mortality in decompensated cirrhotic patients with peptic ulcer hemorrhage.

Mortality occurred in $13.6 \%$ and $19 \%$ of patients with or without antibiotic prophylaxis respectively. Only $20 \%$ of the deaths were directly related to the bleeding episode, whilst the remaining deaths were associated with sepsis ( $n=40,25 \%)$ and multi-organ failure $(n=22,55 \%)$. Despite substantial improvement in PUB management, the mortality in cirrhotic patients remains high, especially among patients with advanced liver cirrhosis [32, 33]. In a recently published study of 10,428 patients with nonvariceal upper gastrointestinal bleeding in non-cirrhotic patients, death was associated with causes directly related to the bleeding episode in only $29 \%$ of cases whilst in the remaining cases, co-morbidity played a fundamental role [34]. In other words, most PUB-linked deaths are not directly caused by the bleeding ulcer itself. This probably explains why routine antibiotic prophylaxis after gastrointestinal bleeding, even though it significantly reduces the incidence of bacterial infections, does not significantly affect the mortality rate. As most mortality occurs as a result of multi-organ failure, this suggests that improved treatments and supportive care should be provided to prevent sepsis complications and key organ failure. On the other hand, three patients died from uncertain causes soon after endoscopy and they were excluded from study. The causes of mortality could not be determined whether they were procedure-related (suffocation, aspiration pneumonia, bowel trauma, incomplete endoscopic hemostasis, as extra), bleeding-related or other causes such as acute myocardial infarction, brain stroke, perforated peptic ulcer, as extra. Therefore, we excluded patients who die within $24 \mathrm{~h}$ due to unable to analyses the cause of mortality.

The Rockall score was found to be an independent predictor of in-hospital mortality [11]. It makes séance as it includes both clinical and endoscopic criteria to predict the risk of rebleeding and death. The bottom line is, we used the MELD score, the Child-Pugh classification, and the Rockall's risk scoring system to assess our patients but mortality was surprisingly not related to the severity of liver dysfunction as expressed by either the MELD score 
or the Child-Pugh classification. Only Rockall scores were associated with rebleeding and death. The effectiveness of the Rockall score as a predictor of in-hospital death in cirrhotic patients with PUB proved to be similar to that reported in non-cirrhotic patients [35].

A major strength of this study is that it was a welldefined consecutive registered study cohort treated by a standard endoscopic intervention. Patients who received antibiotic prophylaxis were defined as antibiotic group and those who not received were used as historical control. To provide the highest possible level of uniformity and to minimize differences in the entry, only patients who received their initial and subsequent treatment in our unit were studied. The robustness of this study is enhanced by the restriction of subjects to patients without an apparent source of infection at first bleeding episode, strict exclusion criteria and the complete follow-up of the cohort. The use of rebleeding, infection and death as the main outcomes provided consistent and objective endpoints in the study. Unlike other studies, which have included patients at differing disease stages without separate analyses, our study did evaluate the impact of liver function on the efficacy of antibiotics.

Current study encountered some limitations. First, the sample size was small and a single center cohort study. Certain selection biases could exist and must be cautious in extrapolating the results. Multicenter data with a larger sample size is mandatory. Second, the authors also served as data abstractors and could have been biased because they were not blinded which was not possible in this retrospective cohort study. Therefore, exposure bias might be considered a limitation of this study. Third, survivor bias may have occurred in which surviving patients who probably had more opportunities to receive intensive care, such as ventilator support, hemodialysis, repeated endoscopic hemostasis and more blood product transfusions.

Those patients who belonged to control group were enrolled between January 1, 2008, and December 31, 2009. The antibiotic group has been routine practice to prescribe prophylactic antibiotics to cirrhotic patients with gastrointestinal bleeding in our hospital since 2010 . This minimized bias in our results, because both cohorts were enrolled according to identical treatment protocol adhering to our hospital policy in our daily practices for PUB population even though during the different period.

\section{Conclusions}

This study confirmed the beneficial effects of antibiotic prophylaxis in patients with cirrhosis following APU with the reduced infections and rebleeding events. The study also suggests that antibiotic prophylaxis could improve survival in decompensated cirrhotic patients following acute PUB. The Rockall score was the strongest predictive factor of in-hospital mortality. Further studies directed to explore ways to improve the overall outcome of cirrhotic patients with PUB are mandatory.

\section{Abbreviations}

PUB: Peptic ulcer bleeding; NSAID: Non-steroid anti-inflammatory drugs; HBsAg: Hepatitis B antigen; Anti-HCV: Hepatitis C antibody; HR: Hazard ratio; EV: Esophageal varices; GV: Gastric varices; MELD: Model for end-stage liver disease; ESRD: End stage renal disease; COPD: Chronic obstructive pulmonary disease.

\section{Competing interests}

The authors declare that they have no competing interests.

\section{Authors' contributions}

WCT participated in the design of the study, coordinated the study, performed the statistical analysis. MTK, SCY and LSL wrote the manuscript. $\mathrm{CNH}, \mathrm{YHK}, \mathrm{CHK}, \mathrm{CML}, \mathrm{CMK}$ and SKC participated in the design of the study and consulted on the statistical analysis. WCT consulted on design of the study and on the interpretation of results. All authors read and approved the final manuscript.

\section{Acknowledgements}

The authors wish to thank Ms. Ching-Yi Lin for her assistance during the preparation of the manuscript.

\section{Author details}

${ }^{1}$ Division of Hepatogastroenterology, Department of Internal Medicine, Kaohsiung Chang Gung Memorial Hospital, Chang Gung University College of Medicine, Kaohsiung, Taiwan. ²Department of Pharmacy, Kaohsiung Chang Gung Memorial Hospital and Chang Gung University; College of Medicine, Kaohsiung, Taiwan. ${ }^{3}$ Division of Hepatogastroenterology, Kaohsiung Chang Gang Memorial Hospital, 123 Ta-Pei Road, Niaosung Hsiang, Kaohsiung City 833, Taiwan.

Received: 11 February 2015 Accepted: 11 May 2015

Published online: 20 May 2015

\section{References}

1. Goulis J, Armonis A, Patch D, Sabin C, Greenslade L, Burroughs AK. Bacterial infection is independently associated with failure to control bleeding in cirrhotic patients with gastrointestinal hemorrhage. Hepatology vol. 1998;27:1207-12.

2. Vivas S, Rodríguez M, Palacio MA, Linares A, Alonso JL, Rodrigo L. Presence of bacterial infection in bleeding cirrhotic patients is independently associated with early mortality and failure to control bleeding. Dig Dis Sci. 2001;46:2752-7.

3. Rimola A, Bory F, Teres J, Perez-Ayuso RM, Arroyo V, Rodes J. Oral, nonabsorbable antibiotics prevent infection in cirrhotics with gastrointestinal hemorrhage. Hepatology. 1985;3:463-7.

4. Hsu YC, Liou JM, Chung CS, Tseng CH, Lin TL, Chen CC, et al. Early risk stratification with simple clinical parameters for cirrhotic patients with acute upper gastrointestinal bleeding. Am J Emerg Med. 2010;28:884-90.

5. D’Amico G, De Franchis R, Cooperative Study Group. Upper digestive bleeding in cirrhosis. Post-therapeutic outcome and prognostic indicators. Hepatology. 2003;38:599-612.

6. Arvaniti V, D'Amico G, Fede G, Manousou P, Tsochatzis E, Pleguezuelo M, et al. Infections in patients with cirrhosis increase mortality four-fold and should be used in determining prognosis. Gastroenterology. 2010;139:1246-56.

7. Garcia-Tsao G, Bosch J. Management of varices and variceal hemorrhage in cirrhosis. N Engl J Med. 2010;362:823-32.

8. Soriano G, Guarner C, Tomas A, Villanueva C, Torras X, González D, et al. Norfloxacin prevents bacterial infection in cirrhotics with gastrointestinal hemorrhage. Gastroenterology. 1992;103:1267-72.

9. Soares-Weiser K, Brezis M, Tur-Kaspa R, Leibovici L. Antibiotic prophylaxis for cirrhotic patients with gastrointestinal bleeding. Cochrane Database Syst Rev. 2002;2. CD002907

10. Forrest JA, Finlayson ND, Shearman DJ. Endoscopy in gastrointestinal bleeding. Lancet. 1974;2:394-7. 
11. Rockall TA, Logan RF, Devlin HB, Northfield TC. Risk assessment after acute upper gastrointestinal haemorrhage. Gut. 1996;38:316-21.

12. Hung CH, Lu SN, Wang JH, Lee CM, Chen CH, Changchien CS. Correlation between ultrasonographic and pathologic diagnoses of hepatitis B and $C$ virus-related cirrhosis. J Gastroenterol. 2003;38:202-3.

13. O'Shea RS, Dasarathy S, McCullough AJ. Alcoholic liver disease. Hepatology. 2010;51:307-28.

14. Lu LS, Lin SC, Kuo CM, Tai WC, Tseng PL, Chang KC, et al. A real world report on intravenous high-dose and non-high-dose proton-pump inhibitors therapy in patients with endoscopically treated high-risk peptic ulcer bleeding. Gastroenterol Res Pract. 2012;2012:858612.

15. Wu CK, Wang JH, Lee CH, Wu KL, Tai WC, Lu SN, et al. The outcome of prophylactic intravenous cefazolin and ceftriaxone in cirrhotic patients at different clinical stages of disease after endoscopic interventions for acute variceal hemorrhage. PLoS One. 2013;8:e61666.

16. Gao B, Seki E, Brenner DA, Friedman S, Cohen Jl, Nagy L, et al. Innate immunity in alcoholic liver disease. Am J Physiol Gastrointest Liver Physiol. 2011;300:G516-25.

17. Haglind E, Wang D, Klein AS. Hepatic reticuloendothelial system dysfunction after intestinal ischemia-reperfusion. Shock. 1996;5:72-5.

18. Kondo S, Wang D, Mayumi T T, Klein AS, Bulkley GB. Effect of hemorrhagic shock and resuscitation upon hepatic phagocytic clearance and killing of circulating micro organisms. Shock. 1996:5:106-11.

19. Borzio M, Salerno F, Piantoni L, Cazzaniga M, Angeli P, Bissoli F, et al. Bacterial infection in patients with advanced cirrhosis: a multicentre prospective study. Dig Liver Dis. 2001;33:41-8.

20. Xu HW, Wang JH, Tsai MS, Wu KL, Chiou SS, Changchien CS, et al. The effects of cefazolin on cirrhotic patients with acute variceal hemorrhage after endoscopic interventions. Surg Endosc. 2011;25:2911-8.

21. Goulis J, Patch D, Burroughs AK. Bacterial infection in the pathogenesis of variceal bleeding. Lancet vol. 1999;353:139-42.

22. Chavez-Tapia NC, Barrientos-Gutierrez T, Tellez-Avila F, Soares-Weiser K, Mendez-Sanchez N, Gluud C, et al. Meta-analysis: antibiotic prophylaxis for cirrhotic patients with upper gastrointestinal bleeding - an updated Cochrane review. Aliment Pharmacol Ther. 2011;34:509-18.

23. Rosa $\mathrm{H}$, Silverio AO, Perini RF, Arruda CB. Bacterial infection in cirrhotic patients and its relationship with alcohol. AmJ Gastroenterol. 2000;95:1290-12930.

24. Wyke RJ. Problems of bacterial infection in patients with liver disease. Gut 1987;28:623-41.

25. Shorvon PJ, Eykyn SJ, Cotton PB. Gastrointestinal instrumentation, bacteremia, and endocarditis. Gut. 1983;24:1078-93.

26. Fernández J, Navasa M, Gómez J, Colmenero J, Vila J, Arroyo V, et al. Bacterial infection in cirrhosis: epidemiological changes with invasive procedures and norfloxacin prophylaxis. Hepatology. 2002;35:140-8.

27. Marmo R, Koch M, Cipolletta L, Capurso L, Pera A, Bianco MA, et al. Predictive factors of mortality from nonvariceal upper gastrointestinal hemorrhage: a multi-center study. Am J Gastroenterol. 2008;103:1639-47.

28. Rudler M, Rousseau G, Benosman H, Massard J, Deforges L, Lebray P, et al. Peptic ulcer bleeding in patients with or without cirrhosis. Aliment Pharmacol Ther. 2012;36:166-72.

29. Venkatesh PG, Parasa S, Njei B, Sanaka MR, Navaneethan U. Gastrointest Endosc. 2014;79:605-14.

30. Lanas A, García-Rodríguez LA, Polo-Tomás M, Ponce M, Alonso-Abreu l, Perez-Aisa MA, et al. Time trends and impact of upper and lower gastrointestinal bleeding and perforation in clinical practice. Am J Gastroenterol. 2009;104:1633-41.

31. Foreman MG, Mannino DM, Moss M. Cirrhosis as a risk factor for sepsis and death: analysis of the National Hospital Discharge Survey. Chest. 2003;124:1016-20.

32. Hsu YC, Lin JT, Chen TT, Wu MS, Wu CY. Long-term risk of recurrent peptic ulcer bleeding in patients with liver cirrhosis: A 10-year nationwide cohort study. Hepatology. 2012;56:698-705.

33. Thabut D, Bernard-Chaber TB. Management of acute bleeding from portal hypertension. Best Pract Res Clin Gastroenterol. 2007;21:19-29.

34. Sung JJ, Tsoi KK, Ma TK, Yung MY, Lau JY, Chiu PW. Causes of mortality in patients with peptic ulcer bleeding: a prospective cohort study of 10,428 cases. Am J Gastroenterol. 2010;105:84-9.

35. Sanders DS, Carter MJ, Goodchap RJ, Cross SS, Gleeson DC, Lobo AJ. Prospective validation of the Rockall risk scoring system for upper $G$ hemorrhage in subgroups of patients with varices and peptic ulcers. Am J Gastroenterol. 2002;97:630-5.

\section{Submit your next manuscript to BioMed Central and take full advantage of:}

- Convenient online submission

- Thorough peer review

- No space constraints or color figure charges

- Immediate publication on acceptance

- Inclusion in PubMed, CAS, Scopus and Google Scholar

- Research which is freely available for redistribution

Submit your manuscript at www.biomedcentral.com/submit 